Cahiers « Mondes anciens »

ANCIENS

Histoire et anthropologie des mondes anciens

15 | 2022

Les parures divines

\title{
Les parures divines
}

Du Proche-Orient à la Méditerranée dans les mondes anciens : quelques pistes de recherche

Divine Ornaments from the Near East to the Mediterranean in Antiquity: Some

Research Issues

Florence Gherchanoc et Louise Quillien

\section{OpenEdition}

Journals

Édition électronique

URL : https://journals.openedition.org/mondesanciens/3567

DOI : 10.4000/mondesanciens.3567

ISSN : 2107-0199

Éditeur

UMR 8210 Anthropologie et Histoire des Mondes Antiques

Référence électronique

Florence Gherchanoc et Louise Quillien, «Les parures divines », Cahiers « Mondes anciens » [En ligne], 15 | 2022, mis en ligne le 18 janvier 2022, consulté le 21 janvier 2022. URL : http://

journals.openedition.org/mondesanciens/3567 ; DOI : https://doi.org/10.4000/mondesanciens.3567

Ce document a été généré automatiquement le 21 janvier 2022.

\section{$(1) \Theta$}

Les Cahiers «Mondes Anciens » sont mis à disposition selon les termes de la licence Creative Commons Attribution - Pas d'Utilisation Commerciale - Pas de Modification 4.0 International. 


\section{Les parures divines}

Du Proche-Orient à la Méditerranée dans les mondes anciens : quelques pistes de recherche

Divine Ornaments from the Near East to the Mediterranean in Antiquity: Some

Research Issues

Florence Gherchanoc et Louise Quillien

1 Ce dossier regroupe les articles présentés lors de la table ronde Divines parures $d u$ Proche-Orient au monde méditerranéen dans l'Antiquité, organisée en juin 2018 à l'INHA (Paris). L'objectif de cette rencontre scientifique était d'ouvrir des pistes de recherche communes au Proche-Orient ancien et au monde gréco-romain, pour l'étude des parures divines. Nous remercions chaleureusement le LabEx HASTEC (Histoire et anthropologie des sciences, des techniques et des croyances) et le laboratoire ANHIMA (Anthropologie et histoire des mondes anciens, UMR 8210) d'avoir permis, par leur soutien, la réalisation de cette table ronde et sa publication.

\section{Les parures divines, définition}

2 Le terme "parure ", dans les mondes anciens, recouvre des réalités variées, comme l'ont montré Lydie Bodiou, Florence Gherchanoc, Valérie Huet et Véronique Mehl dans leur ouvrage Parures et artifices: le corps exposé dans l'Antiquité, Paris (2011) ${ }^{1}$. Comme de nos jours, il caractérise « ce qui embellit, met en valeur quelque chose ». En français, il désigne aussi plus précisément une "garniture de pierreries ou de perles comprenant collier, bracelets, etc. », et " un ensemble de [sous-]vêtement féminins »².

Ce terme désigne ainsi tout ce qui modifie l'apparence et est destiné à l'ornementation du corps. Ce dispositif matériel (tout produit, tout accessoire) visible, parfois olfactif et sonore, est « soit apposé sur le vêtement, directement sur le corps vivant ou mort [ou encore figuré, statufié], [soit disposé] dans son environnement proche, ou bien encore destiné à être manipulé, [actionné] pour mettre en valeur une gestuelle spécifique », un mouvement ${ }^{3}$. Suivant cette définition, les articles ici rassemblés étudient non seulement les bijoux, mais également les coiffes et coiffures, les vêtements, les 
cosmétiques et les miroirs. Ils abordent aussi la dimension olfactive (Laura Cousin) et musicale (Eleonora Colangelo) des parures. Ils soulignent ainsi comment, tout comme les vêtements, les parures ont un sens, suivent des codes et transmettent des informations. Celles-ci, en effet, peuvent véhiculer des messages dans les registres de la croyance, de l'identité et du pouvoir.

4 Parer les dieux, leurs effigies, relève d'une mise en ordre définie le plus souvent par des règlements cultuels respectant la volonté des divinités. Ainsi, le roi assyrien Assarhaddon (vII siècle av. $n$. è.) écrit-il au sujet des réparations des statues des dieux babyloniens :

Avec des ornements splendides et des bijoux précieux, j'ai paré leurs cous et recouvert leurs poitrines, exactement comme le grand seigneur, le dieu Marduk, le souhaitait et comme il plaisait à la reine, la déesse Șarpanitu4 .

5 En grec, le terme katakosmêsis renvoie à ce type de processus d'arrangement et de soin, à l'action de parer les statues cultuelles. Les parures divines participent de ces opérations techniques et traitements spécifiques ainsi que des mises en scène élaborées pour présentifier la divinité dans toute sa puissance. Parfums et vêtements en constituent des éléments particuliers ${ }^{5}$, conformément (kata kosmon) à ce qui est attendu. À cet égard, l'épisode raconté par Plutarque à propos du peplos offert à Athéna lors des Panathénées, de l'impiété des Athéniens suite à un décret de Stratoclès de 307-306 av. n. è. et de l'hubris de l'Antigonide Démétrios est significatif :

Les Athéniens furent les seuls à les proclamer dieux sauveurs [...]. Ils décidèrent aussi que les images d'Antigone et de Démétrios seraient tissées parmi celles des dieux sur le peplos. [...] La divinité signifia son sentiment sur la plupart de ces mesures. Le peplos, où l'on avait décidé de tisser les images de Démétrios et d'Antigone auprès de celles de Zeus et d'Athéna, pendant qu'on le promenait à travers le Céramique, fut déchiré en deux par une bourrasque ${ }^{6}$.

6 Autrement dit, ce peplos destiné à Athéna n'est pas une parure conforme. Le choix de faire figurer sur l'étoffe divine les deux Antigonides constitue une forme d'impiété. Il ne répond pas à une bonne mise en ordre.

Nous pouvons par conséquent qualifier de parures «divines" les parures qui sont la propriété d'une divinité et conformes à ses attributs ainsi qu'au contexte et aux gestes rituels propres au culte du dieu qui accompagnent l'acte de parer, orner ${ }^{7}$, que ces parures soient portées par son effigie ou bien conservées dans le trésor de son sanctuaire. De plus, ces parures ont-elles un sens particulier lorsqu'elles appartiennent à une divinité ? En d'autres termes, quelles sont les spécificités des parures de divinités ou des divines parures - cela ne dit-il pas la même chose ? Dans le premier cas, il s'agit de s'interroger sur leurs traits communs du Proche-Orient à la Méditerranée, en considérant leur matérialité, la nature, la qualité et l'agencement des matériaux qui les composent - des tissus (lin, laine), des bijoux et autres ornements en bois, or, pierres précieuses -, en étudiant leur éclat et leur couleur (blanc, safran, pourpre, etc.), en s'attachant à leur facture et à la technicité. Ainsi, il est possible de réfléchir à la valeur de ces parures divines, valeur concrète en termes de prix et valeur symbolique en lien avec leur statut et leur place dans un rituel ou une célébration donnée. Dans le second cas, la question porte sur ce qui qualifie une divine parure. Celle-ci est-elle chargée d'une valeur sacrée, d'un pouvoir particulier et par quel processus technique et/ou rituel ? Ces objets sont, en effet, destinés à intégrer un lieu de culte et, pour certains d'entre eux, à entrer en contact avec le corps d'un dieu ou d'une déesse. Ils partagent donc quelque chose avec le divin. S'agit-il de qualités intrinsèques ou bien acquises, soit 
par la manière dont ils ont été fabriqués, soit par un processus d'offrande ou de consécration? En outre, une divine parure est-elle obligatoirement indissociable de l'image divine à laquelle on la consacre ? Peut-elle devenir autonome de la divinité et de son effigie ? Peut-elle être dotée d'une agentivité, d'une puissance qui lui est propre ou encore faire l'objet d'un culte spécifique? Enfin, quels sont les liens entre une divinité, sa nature, son mode d'action et sa parure ? Les études réunies dans ce dossier apportent dans la mesure du possible différentes réponses à ces questions, en fonction du contexte géographique et historique considéré ainsi que de la parure et de la divinité envisagées.

8 Nous avons choisi de prendre en considération les divinités dans la diversité de leurs présentifications et manifestations: statues, images, personnages littéraires et mythologiques. Les parures divines n'ont pas nécessairement la même fonction dans ces différents contextes. Une statue divine, par exemple, n'a peut-être pas la même sacralité qu'une représentation sur un bas-relief ou un sceau cylindre. Nous renvoyons, pour ces questions, à la riche bibliographie portant sur la représentation du divin dans l'Antiquité, notamment aux travaux fondateurs de Jean-Pierre Vernant ${ }^{8}$.

Dans certaines situations, nous pouvons utiliser l'expression « divines parures », pour parler d'objets de parure qui sont eux-mêmes divinisés ou qui concentrent une partie des pouvoirs divins. Les tiares des dieux babyloniens, la ceinture d'Aphrodite ou encore les gemmes romaines gravées au nom d'une divinité sont autant d'exemples de la sorte, étudiés dans ces différents articles.

Des sources de différentes natures ont été examinées. Elles comprennent, d'une part, les objets archéologiques et les représentations figurées - telles que la stèle représentant la déesse Ninhursag, la statuette de déesse au vase jaillissant du palais de Mari et les pierres gravées romaines - et d'autre part, la documentation textuelle. Parmi les sources textuelles étudiées par les auteurs des articles rassemblés ici, l'on compte les documents administratifs des temples, des palais et des cités (archives de Mari enregistrant des transferts d'objets d'un service du palais à un autre, listes de bijoux des temples néo-babyloniens, inventaires du trésor du sanctuaire de Délos), les œuvres littéraires (inscriptions royales assyriennes et mésopotamiennes, œuvres d'auteurs classiques à l'instar d'Euripide), les textes religieux (rituels babyloniens) et techniques (manuels des lapidaires romains).

11 Les termes qui désignent les parures divines dans les langues anciennes témoignent de la manière dont ces objets étaient définis, catégorisés et perçus. En akkadien, le terme šukuttu, provenant du verbe šakānu (placer) et équivalent du sumérien "gil-sa», désigne les bijoux'. Il n'est pas spécifique au monde divin et s'applique aussi bien à des ornements destinés aux êtres humains. Les inventaires de šukuttu ne comprennent que les bijoux. L'habillement et les parfums sont enregistrés dans d'autres documents. Les bijoux sont décrits en akkadien par un riche vocabulaire qui a évolué dans le temps, tout comme l'ensemble de la langue. À titre d'exemple, à l'époque néo-babylonienne, un large panel de termes est employé pour les décrire. Il existe des termes pour désigner chaque ornement - collier (kišādu), bracelet (semeru), boucles d'oreilles (inșabtu), anneau (unqu), couronne (kulūlu, agû)-, chaque partie d'ornement pendentifs (disque solaire šamšu), cordons (riksu), montures (mandìtu) -, ainsi que pour nommer les pierres, les perles et les sequins classés en fonction de leur matière et de leur forme (datte uHinnu, grenade nurmû, rosette ayyaru, etc.). Cet inventaire n'est qu'un aperçu de la diversité de ce vocabulaire. Certains termes akkadiens sont encore 
difficiles à traduire. L'étude des parures divines permet ainsi de préciser leur définition, par un examen de leur contexte d'apparition dans les textes. À titre d'exemple, les articles qui suivent étudient la nature de l'ornement kukilu à l'époque paléo-babylonienne (Manon Ramez), du pectoral irtu (Louise Quillien) et du pot à cosmétiques mušālu (Laura Cousin) à l'époque néo-babylonienne.

Les textes littéraires emploient également d'autres termes relatifs aux parures. Le mot akkadien tiqnu, qui désigne un équipement ordonné, un bijou, ornement ou insigne, est employé pour qualifier les parures des divinités ${ }^{10}$. Il provient du verbe taqānu, qui signifie au sens premier "devenir placide, en ordre, sûr " et, avec une conjugaison spécifique, tuqqunu, "orner». Cet extrait d'une inscription du roi Assyrien Assarhaddon en illustre l'usage : «J'ai orné (utaqqin-ma) leurs cous [ceux des divinités], j'ai couvert leurs poitrines avec des ornements (tiqni) sublimes, des bijoux (šukuttu) précieux, seyant admirablement à leur royauté ${ }^{11}$.» Moins fréquemment, le verbe akkadien asāmu, "être approprié, correct, adapté12 ${ }^{2}$, équivalent du sumérien $\mathrm{du}_{7}$, est employé par les rois dans leurs inscriptions pour souligner la qualité de leurs offrandes. De ce verbe dérive l'akkadien simtu (en sumérien me-te), qui désigne "ce qui est approprié » et peut qualifier, entre autres, un ornement propre à une divinité13

Le vocabulaire que nous venons de présenter n'est pas spécifique aux parures des divinités. En revanche, il existe une notion particulière aux dieux et déesses, qui décrit leur apparence extérieure à laquelle participent les parures: l'aura, melammu en akkadien ou me-lam ${ }_{2}$ en sumérien. Cette aura se manifeste par un mélange de splendeur, de luminosité et de bonne odeur qui inspire à la fois l'admiration et la terreur ${ }^{14}$. Or, le melammu peut émaner des parures portées par les divinités, comme le montre cet extrait de l'inscription d'Assarhaddon citée plus haut (RINAP 4, 48:85): « cette couronne (aĝ) [pour le dieu Aššur], revêtue du melammu ». Les vêtements divins participent également à l'expression de cette aura, en particulier ceux qui sont portés sur la tête ${ }^{15}$. Les parures des divinités contribuent donc à rendre visible l'aura surnaturelle qui les caractérise.

En grec, le terme kosmos, bien étudié par Michel Casevitz, est celui qui équivaut le mieux à ce que nous entendons par parure. S'il qualifie généralement des atours féminins et leur bon agencement ou ordonnancement, il s'applique également à d'autres parures, y compris celles d'hommes ${ }^{16}$. Il caractérise/désigne un ou plusieurs éléments (sur)ajoutés qui par leur mise en ordre créé(nt) de la beauté - cet ajout recélant, en outre, une part d'artifice, « une œuvre de mêtis, d'intelligence rusée ${ }^{17}$ ", par l'utilisation d'une technê. En latin, comme l'a notamment explicité Sylvia Estienne, le « vocabulaire de la parure, [en partant] en particulier du verbe ornare et des substantifs qui s'y rattachent, ornatio, ornamentum et ornatus ", fait référence à des notions similaires. En effet :

[o]rnare se rattache à la même racine qu'ordo, "le bon ordre“, et renvoyait à l'origine à l'idée de mettre en ordre, d'organiser ; on peut en retenir deux sens principaux : 1. "équiper, préparer", 2. "orner, parer". Le sens le plus ancien d'ornamentum semble être celui d'"équipement", sans connotation esthétique. [...] Ces deux termes peuvent donc désigner des parures corporelles, qui ne se réduisent pas pour autant au simple mundus féminin. Celles-ci ne sont pas en effet perçues pour leur seule valeur ornementale, mais également comme des signes visant à rendre lisible le statut de la personne qui en bénéficie ${ }^{18}$. les parures divines jouent un rôle majeur et particulier dans la mise en ordre et 
l'agencement des attributs divins qu'il conviendra d'interroger; elles sont potentiellement chargées d'une puissance inhérente à leur propriétaire. Et comme le suggère Sylvia Estienne, à propos du monde romain, « l'examen de l'ornatus des dieux et de leurs ornamenta permet de mieux cerner la nature et la fonction des parures divines ${ }^{19}$."

\section{Historiographie}

Parmi les innombrables travaux sur la religion et le culte en Mésopotamie, certains comprennent des éditions de textes ou des analyses concernant les parures des divinités. Il s'agit essentiellement d'études portant sur les textes administratifs des palais et des temples qui documentent leur fabrication, ainsi que sur les inventaires des trésors des temples. De tels documents existent depuis le $\mathrm{III}^{\mathrm{e}}$ millénaire av. n. è. Les archives d'Iri-Saĝrig, datées de l'époque d'Ur III, comprennent des listes d'objets destinés au culte et à l'ornementation des statues divines (Owen 2019). Les « archives du trésor ", également d'époque d'Ur III, renferment des inventaires de biens appartenant à des divinités (Limet 1960 et Paoletti 2012). En ce qui concerne le $\mathrm{II}^{\mathrm{e}}$ millénaire av. n. è., il existe des études sur les bijoux des divinités aux époques paléo-assyrienne (Michel 2006) et paléo-babylonienne (Maggio 2012, Arkhipov 2012). Des travaux ont également été consacrés à l'édition des inventaires de trésors de temples, textes qui mentionnent des vêtements et des bijoux. On compte parmi eux les inventaires de bijoux offerts à la déesse Nin-égal à Qatna (Bottéro 1949; Fales 2004 ; Muller 2015), ceux de la déesse Ishtar de Lagaba (Leemans 1952), ainsi que les inventaires des objets précieux du temple d'Adad à Mê-Turan (Black et Al-Rawi 1983) et du temple d'Eštar à Harrâdum (Joannès et al. 1983).

Il existe également des dossiers d'archives sur les parures des divinités datés du I ${ }^{\text {er }}$ millénaire av. n. è. Les travaux portant sur l'époque néo-assyrienne concernent essentiellement les bijoux des rois et des reines, pour lesquels les sources textuelles, archéologiques et iconographiques sont nombreuses. Cependant, l'étude de Salvatore Gaspa (2018) sur les textiles évoque également les vêtements des divinités. Concernant l'époque néo-babylonienne, les inventaires des trésors des temples d'Uruk et de Sippar ont été étudiés respectivement par Paul-Alain Beaulieu (1999) et Francis Joannès (1992). Les textes administratifs concernant la fabrication des bijoux et des vêtements des dieux dans les temples ont fait l'objet de plusieurs travaux: Sack 1977; Bongenaar 1997 ; Beaulieu 2003 ; Zawadzki 1985, 1991, 2006 et 2013. Des études plus spécifiques ont été réalisées sur les vêtements ornés d'or, qu'ils soient attribués aux divinités ou bien aux membres de l'élite babylonienne et assyrienne: Oppenheim 1949, Barrelet 1977, Parpola 1985, Gaspa 2014 et Joannès 2020. Nous ne détaillerons pas ici l'ensemble des travaux portant sur l'orfèvrerie et la joaillerie en Mésopotamie, car ils ne concernent pas spécifiquement les parures divines. Cet exposé des travaux antérieurs montre que l'ornementation des divinités est une pratique ancienne et bien répandue en Mésopotamie. Ces études permettent d'avoir à disposition un grand nombre de sources, auxquelles s'ajouteront des fonds encore inédits.

Pour les mondes grec et romain, ces dernières années, les recherches sur les corps des dieux et la figuration du divin se sont inscrites dans la lignée des travaux de J.P. Vernant et des membres du centre Louis Gernet ${ }^{20}$ comme le démontrent, en premier lieu, les résultats des analyses menées par des groupes de recherche internationaux 
comme «Imago : Image et religion » et « Figura » et celles de chercheurs sur la notion $d^{\prime}$ ' image cultuelle $»^{21}$. Cependant, si les réflexions ont été riches sur la question de l'objectivation du corps des dieux et sur celle de la présence divine, elles ne se sont pas toujours focalisées précisément sur les parures divines. De fait, cette interrogation a été développée, plus spécifiquement, dans le cadre du programme de recherche consacré aux associations entre corps, gestes et vêtement, co-animé par Florence Gherchanoc et Valérie Huet, respectivement à ANHIMA et au CRBC. Sous cet angle, en juin 2012, lors d'une journée d'étude, des contributions se sont attachées aux parures divines, aux liens entre la divinité, sa nature, son mode d'action et ses atours : au voile qui souligne les prérogatives d'Aphrodite en matière de séduction et en mer, ou encore aux jeux de vêtement pour présentifier les dieux ${ }^{22}$. L'ensemble a montré comment les parures construisent la présentification du dieu, énoncent dans différents types de discours et en contexte rituel son identité et des éléments de sa puissance ${ }^{23}$. À cet égard, bien que focalisé sur les objets, suivant les perspectives d'un autre programme de recherche, l'article, co-écrit par Manon Brouillet et Cléo Carastro, consacré au kestos d'Aphrodite et à l'égide d'Athéna, est extrêmement précieux ; les autrices s'interrogent, en effet, sur la manière dont le texte homérique construit ces deux artefacts relevant de la sphère divine; s'attachant moins à leur apparence et à leur matière qu'à leurs composantes, elles montrent comment ces parures divines se trouvent être des puissances ${ }^{24}$. D'autres études ont porté, elles, précisément, sur le geste de parer les divinités, les effigies divines ou encore des stèles, dans les rituels et cultes des dieux, posant la question de la signification des gestes, de la visibilité et de la manipulation rituelle ${ }^{25}$.

\section{Problématiques et pistes de recherche}

Les études rassemblées dans ce volume apportent des éléments supplémentaires pour mieux comprendre quel était le rôle des parures divines dans les croyances et les pratiques religieuses des anciens. Offertes aux divinités, sont-elles des instruments de dévotion, des ressources économiques, des supports de croyances et de pratiques? Placées sur le corps ou à proximité d'une représentation divine, comment participentelles à l'incarnation des dieux dans un objet et comment expriment-elles leurs pouvoirs? De plus, ces parures circulent et acquièrent une certaine autonomie. Dès lors comment se transmettent-elles et quels sont les effets de leur puissance pour ceux qui les possèdent? Trois grandes thématiques ont été abordées dans les articles de ce dossier et permettent d'apporter des réponses à ces questions.

\section{La fabrication et l'aspect matériel des parures divines}

La première thématique porte sur l'aspect matériel des parures divines. Quelles sont leurs modalités de fabrication? Qui en sont les commanditaires et comment travaillent les artisans chargés de leur confection? Dans le royaume de Mari (Syrie), au $\mathrm{II}^{\mathrm{e}}$ millénaire av. n. è., tout comme dans l'Assyrie et la Babylonie du I ${ }^{\mathrm{er}}$ millénaire av. n. è., les rois sont les premiers commanditaires des parures destinées aux divinités. Ils manifestent ainsi leur dévotion, leur pouvoir et leur rôle de vicaire des dieux. Tel un mécène, le roi de Mari fournit les matériaux précieux nécessaires à leur fabrication (Manon Ramez). Au I ${ }^{\text {er }}$ millénaire av. n. è., les reines ne sont pas en reste et sont également d'importantes donatrices (Laura Cousin). À cette époque, les rois babyloniens et assyriens, selon le discours idéologique véhiculé par les inscriptions 
royales, fournissent les matériaux et nomment les artisans chargés de les fabriquer (Louise Quillien). En communiquant avec les dieux par des oracles, les rois les désignent comme les véritables commanditaires de leurs parures et les seuls maîtres de l'aspect que doit avoir leur incarnation sur terre. Dans le monde grec à l'époque classique, le rôle des individus, des groupes intermédiaires et des cités en tant que commanditaires et donateurs d'ornements destinés aux divinités est manifeste (Clarisse Prêtre). La question des intentions se pose de la même manière qu'en Mésopotamie : piété envers la divinité ou glorification du donateur ? Cependant, le don individuel est moins visible en Mésopotamie, où les taxes et les offrandes apportées par la population aux temples semblent servir de réserve financière.

Qui sont les artisans chargés de fabriquer les parures des dieux? Les inscriptions royales assyriennes et babyloniennes exaltent le rôle des artisans et leur savoir d'experts. Ils sont décrits comme les mains des dieux. Les archives administratives, dites « de la pratique », donnent à voir le travail concret et quotidien des joailliers, des orfèvres, des tisserands. Ils ont des statuts variés, des savoir-faire spécialisés, et sont plus souvent occupés à réparer les objets de parure qu'à en fabriquer de nouveaux (Manon Ramez, Louise Quillien). Dans le monde grec, à l'époque classique, il ne semble pas exister d'artisanat dédié à la fabrication des parures des divinités sur une base régulière. Certains objets sont donnés après avoir servi et ont une valeur affective. D'autres sont créés spécialement pour la divinité (Clarisse Prêtre). Dans le monde romain, l'exemple des lapidaires témoigne de l'importance du savoir-faire des artisans, à la fois technique et religieux. La gravure d'une pierre est un rite qui la dote d'une puissance divine, fabrication et consécration sont alors mêlées (Thomas Galoppin). Dans tous les cas, les anciens accordent aux divinités un rôle dans la fabrication de leurs parures.

Quels sont les matériaux employés pour les fabriquer et quelles sont leurs formes? Tous les objets ne peuvent pas entrer dans le sanctuaire et orner la divinité. De l'importance peut être donnée au matériau, à la facture, à la forme, ou bien seulement au geste de l'offrande ou de la consécration. À partir des représentations iconographiques et des vestiges archéologiques, il est possible de reconstituer l'aspect matériel des parures, comme cela a été démontré pour les coiffes des divinités pré et proto-dynastiques mésopotamiennes (Catherine Breniquet). Ces parures étaient composées d'éléments animaux, végétaux et humains. Ce caractère composite est également manifeste dans le monde grec (Clarisse Prêtre). Dans les royaumes mésopotamiens des $\mathrm{II}^{\mathrm{e}}$ et $\mathrm{I}^{\mathrm{er}}$ millénaire av. n. è., les matériaux utilisés pour leur fabrication sont le plus souvent précieux: or, argent, pierres fines (Manon Ramez, Laura Cousin). On attribue à ces matériaux des qualités particulières qui les rendent aptes à être destinés aux dieux. En Mésopotamie comme dans le monde romain, par exemple, des propriétés et des pouvoirs sont attribués aux pierres (Thomas Galoppin). Mais le prix et la matière des parures dépendent aussi des capacités financières des commanditaires, des donateurs et des sanctuaires. Les inventaires de Délos mentionnent des offrandes individuelles de qualités diverses, chacun offrant selon ses moyens. Des statues portent ainsi des bijoux en plaqué or. Au-delà de l'aspect matériel des parures, les textes littéraires grecs permettent, en les décrivant en action, d'en saisir tout l'effet sensoriel, du visuel à l'olfactif, en passant par la sonorité ou musicalité (Eleonora Colangelo). 


\section{Les relations entre les parures et les divinités qui les possèdent et qui les portent}

deuxième thématique concerne les relations entre les parures et les divinités. Tout d'abord, quels sont les liens entre le corps du dieu et ses parures? Les coiffes des premières divinités mésopotamiennes sont un prolongement du corps des statues divines, jusqu'à en faire partie. Les cornes de vache, emblèmes des divinités en Mésopotamie, qui composent ces coiffes, viennent s'enchâsser sur la tête comme si elles en sortaient (Catherine Breniquet). Une distinction doit cependant être faite entre les ornements fixes et ceux qui sont mobiles (Manon Ramez). En Babylonie, au $\mathrm{I}^{\mathrm{er}}$ millénaire av. n. è., les statues divines étaient couvertes de plusieurs strates de vêtements et de dizaines de bijoux, et l'aspect extérieur des divinités devait davantage à ces parures qu'à l'âme en bois des statues (Louise Quillien). En Mésopotamie comme en Grèce, la diversité infinie des objets inventoriés dans les comptes des sanctuaires indique que de nombreuses parties du corps des dieux pouvaient être ornées, même si certaines parures avaient vocation à être offertes aux dieux sans nécessairement être portées par leurs effigies (Clarisse Prêtre).

24 Existe-t-il une hiérarchie entre les parures? L'importance des parures de tête, qui expriment un statut, une identité personnelle, qui sont à la fois vecteur de puissance et de protection, est manifeste en Mésopotamie (Catherine Breniquet). Nous pouvons constater à la fois l'existence d'ornements représentatifs d'une divinité en particulier et qui permettent de l'identifier, et d'autres dont les motifs et symboles ne leur sont pas spécifiques voire circulent entre divinités et même hors du cercle divin (Louise Quillien, Thomas Galoppin). Les sources grecques mettent en exergue l'importance de la parure pour présentifier le dieu, tout en jouant des ambivalences entre divinités et des ressemblances des unes aux autres; elles mettent également en avant l'importance de la manière d'agencer les parures, de les mettre en ordre, action incarnée par le dieu Éros dans des tragédies d'Euripide (Eleonora Colangelo). Ces règles transparaissent également dans les rituels mésopotamiens.

Il est apparu que certains ornements étaient réservés à un genre en particulier, par exemple le kulïlu, uniquement porté par les déesses à Mari (Manon Ramez). Si les parfums et cosmétiques sont portés par les femmes et par les hommes en Mésopotamie, les miroirs, peignes et pots à fards sont emblématiques de la féminité et figurent parmi les accessoires des déesses, des reines et des femmes de l'élite (Laura Cousin). En Grèce, miroirs et cosmétiques sont aussi des attributs féminins, bien que ce soit un dieu, Éros, qui gouverne l'action de se parer (Eleonora Colangelo). En Mésopotamie comme dans le monde gréco-romain, certaines divinités transgressent les frontières du genre, notamment par leurs vêtements, à l'image d'Ištar en Mésopotamie ou d'Athéna dans le monde grec. Certaines parures divines ont en elles ces pouvoirs transgressifs: on attribue à la parure d'Aphrodite, à l'époque romaine, le pouvoir de féminiser l'homme qui la porte (Thomas Galoppin).

Comment les parures sont-elles consacrées, offertes et placées sur le corps de la divinité ? L'action de parer une divinité est peu documentée en Mésopotamie. Les parures semblent être manipulées par des prêtres et conservées dans des lieux bien gardés. L'action de parer la statue d'un sanctuaire est rarement décrite dans les textes épigraphiques grecs, qui évoquent surtout les rites d'onction (Clarisse Prêtre). En revanche, des textes littéraires comme la tragédie d'Euripide décrivent les dieux en 
train de se parer (Eleonora Colangelo). Ils illustrent également l'aspect performatif des gestes accomplis avec les parures: par exemple Aphrodite détachant son ruban (Thomas Galoppin).

\section{Les pouvoirs des parures divines}

27 La troisième thématique porte sur les pouvoirs des parures divines. Ces parures ont des significations symboliques et identitaires, mais qui sont bien souvent difficiles à saisir, en l'absence de textes explicites. Ainsi, les cornes, attributs des divinités en Mésopotamie, ont tantôt été interprétées comme un symbole de la force virile, tantôt comme celui de la vache nourricière (Catherine Breniquet). Les parures peuvent également être polysémiques. Les coiffes des premières divinités mésopotamiennes associent par exemple des éléments humains et animaux, des attributs masculins et féminins. Elles sont paradoxales, comme l'est la divinité elle-même, être surnaturel incarné dans un corps fait de matière inanimée.

Certaines parures se voient attribuer des pouvoirs. C'est le cas des pierres et amulettes apotropaïques en Mésopotamie, comme dans le monde gréco-romain (Thomas Galoppin). D'autres concentrent l'entièreté de la puissance divine. Sur leur tête, les premières divinités mésopotamiennes portaient une coiffe dont le cimier figurait un visage humain (Catherine Breniquet). S'agissait-il d'une autre divinité dans la divinité ou d'une concentration de la puissance divine dans la parure de tête? Le chatoiement des bijoux en or et en pierres semi-précieuses et des vêtements ornés de sequins devait manifester, en Mésopotamie, l'aura melammu, à la fois splendide et terrifiante, qui émanait des divinités (Louise Quillien). Les parures donnent ainsi à voir la puissance divine. Cet aspect théâtral et performatif est bien visible, à l'époque classique, dans les tragédies d'Euripide (Eleonora Colangelo). Que serait le dieu sans ses parures? En Mésopotamie, l'un et l'autre semblent dépendants. À Mari, la statue d'une déesse n'est activée qu'à partir du moment où elle porte ses ornements (Manon Ramez) et les récits mythologiques racontent qu'un dieu qui perd sa tiare voit s'envoler sa puissance (Louise Quillien).

De manière plus prosaïque, les parures, en tant qu'objets précieux, sont également des enjeux économiques et de pouvoir. Elles constituent une réserve financière pour les sanctuaires mésopotamiens et grecs et un pouvoir pour ceux qui s'arrogent des droits sur ces finances (Manon Ramez, Clarisse Prêtre). L'on comprend donc pourquoi nous avons retrouvé tant d'inventaires de ces précieuses ressources, en Mésopotamie comme dans le monde gréco-romain. Les dons aux temples ont une fonction à la fois religieuse, politique et économique.

Il existe des similitudes entre les parures luxueuses arborées par les divinités et celles des rois et reines, voir des hauts fonctionnaires palatiaux (Laura Cousin). Pour les rois et l'élite, l'appropriation de certains modèles issus du domaine religieux permet de rehausser leur propre pouvoir. Les amulettes porteuses de puissance divine, arborées par des êtres humains, leur permettent de s'approprier une parcelle des pouvoirs divins (Thomas Galoppin). Certains rois vont même jusqu'à revêtir, dans les textes littéraires mésopotamiens, l'aura melammu des dieux. Cependant, les parures de tête, la tiare à cornes en Mésopotamie, demeurent les attributs non équivoques des divinités. 


\section{De l'Orient à la Méditerranée}

31 Les différentes études présentées dans ce dossier ont permis de mettre en lumière à la fois des traits communs et des différences entre les deux aires géographiques et temporelles considérées. Il existe une différence majeure due aux modèles d'organisation politique, en Mésopotamie, la royauté et en Grèce aux époques classique et hellénistique, la cité et les royaumes. Ainsi, en Mésopotamie, les parures sont commanditées par les rois, et les temples disposent d'un artisanat dédié. Dans le monde grec, elles sont offertes aux divinités par des particuliers, certaines en tant qu'ex-voto, mais aussi par des chefs politiques et militaires comme par des cités, soit à titre personnel, soit lors de fêtes spécifiques. Les valeurs associées au luxe et aux objets précieux sont également différentes.

En dépit de tout cela, les parures des divinités ont parfois des fonctions similaires dans ces deux espaces. Elles ont la même valeur économique et représentent le même enjeu de pouvoir pour les donateurs privés ou institutionnels et les sanctuaires. Sur le plan de la représentation du divin, on constate une même incarnation des divinités dans des objets, notamment dans les statues anthropomorphes, et une même propension à offrir à ces dieux des ornements. Que ce soit dans les rituels ou dans le théâtre, les dieux sont mis en scène avec leurs parures ou en train d'être parés. Enfin, il existe un même lien entre la parure et la manifestation de la puissance divine. Le divin s'incarne à travers des objets, par un processus qui part de l'acte technique de la fabrication, qui se poursuit par l'activation au moyen d'offrande ou de consécration, et qui se termine par les gestes rituels accomplis avec l'objet. Lorsque les objets de parure, loin d'être accessoires, sont investis de tout ou partie des pouvoirs divins, nous voyons à l'œuvre le processus d'incarnation du divin dans une apparence matérielle, qui apparaît comme vivante et surnaturelle. L'étude des parures divines est donc un moyen d'appréhender le rôle de la matérialité dans les croyances et les pratiques religieuses.

\section{BIBLIOGRAPHIE}

\section{Abréviations}

AHw : von Soden W., Akkadisches Handwörterbuch, Wiesbaden, 1965-1981 (3 vol.).

CAD : Roth M. T. dir., The Assyrian Dictionary of the Oriental Institute of the University of Chicago, Winona Lake, 1956-2010 (26 vol.). 


\section{Bibliographie générale}

Arkhipov I. (2012), Le vocabulaire de la métallurgie et la nomenclature des objets en métal dans les textes de Mari, Matériaux pour le Dictionnaire de Babylonien de Paris III, ARM 32, Louvain-ParisWalpole.

Asher-Greve J. M. et Westenholz J. G. (2013), Goddesses in Context: On Divine Powers, Roles, Relationships and Gender in Mesopotamian Textual and Visual Sources, Orbis Biblicus et Orientalis 259, Fribourg-Göttingen.

Barrelet M.-T. (1977), « Un inventaire de Kar-Tukulti-Ninurta : textiles décorés assyriens et autres ", Revue d'assyriologie et d'archéologie orientale 71-1, p. 51-92.

Beaulieu P.-A. (1999), « Un inventaire de joaillerie sacrée de l'Eanna d'Uruk », Revue d'assyriologie et d'archéologie orientale 93-2, p. 141-155.

Beaulieu P.-A. (2003), The Pantheon of Uruk during the Neo-Babylonian Period, Cuneiform Monographs 23, Leyde-Boston.

Belayche N. et Pirenne-Delforge V. éd. (2015), Fabriquer du divin. Construction et ajustements de la représentation des dieux dans l'Antiquité, Liège.

Bettinetti S. (2001), La statua di culto nella pratica rituale greca, Bari.

Black J. A. et Al-Rawi F.N.H. (1983), « The Jewels of Adad », SUMER 39, p. 137-143.

Bodiou L., Gherchanoc F., Huet V. et Mehl V. dir. (2011), Parures et artifices : le corps exposé dans l'Antiquité, Paris.

Bodiou L. et Mehl. V. (2019), « Parure », dans Ead. dir., Dictionnaire du corps dans l'Antiquité, Rennes, p. 469-471.

Bongenaar A.C.V.M. (1997), The Neo-Babylonian Ebabbar Temple at Sippar: Its Administration and Its Prosopography, Publications de l'Institut historique-archéologique néerlandais de Stamboul 80, Leyde.

Borgeaud P. et Fabiano D. éd. (2013), Perception et construction du divin dans l'Antiquité, Genève.

Borger R. (1956), Die Inschriften Asarhaddons, Königs von Assyrien, AfO Beiheft 9, Osnabrück.

Bottéro J. (1949), « Les inventaires de Qatna », Revue d'assyriologie et d'archéologie orientale 43-1/2, p. 1-40.

Briand M. (2011), « De la parure à l'harmonie du monde : esthétique et idéologie du kosmos dans la poésie mélique grecque archaïque », dans Bodiou L., Gherchanoc F., Huet V., Mehl V. dir., Parures et artifices : le corps exposé dans l'Antiquité, Paris, p. 217-232.

Brouillet M. et Carastro C. (2018), « Parures divines. Puissances et constructions homériques de l'objet ", dans Brouillet M. et Carastro C. éd., Place aux objets! Présentification et vie des artefacts en Grèce ancienne, Mètis N.S.16, p. 85-106.

Cartledge P., Millet P. et von Reden S. éd. (1998), Kosmos, Essays in Order, Conflict and Community in Classical Athens, Cambridge.

Casevitz M. (1989), «À la recherche du Kosmos », Le monde, Le temps de la réflexion X, p. 97-119.

Cassin E. (1968), La splendeur divine. Introduction à l'étude de la mentalité mésopotamienne, Paris-La Haye. 
Colburn C. S. et Heyn M. K. (2008), « Introduction. Bodily Adornment and Identity », dans Ead. éd., Reading a Dynamic Canvas. Adornment in the Ancient Mediterranean World, Cambridge, p. 1-12.

Dugast S., Jaillard D. et Manfrini I. éd. (2021), Agalma ou les figurations de l'invisible. Approches comparées, Grenoble.

Estienne S. (2014), «'Parer' les dieux à Rome : ornatio, ornamenta et ornatus deorum », dans Huet V. et Gherchanoc F. dir., De la théâtralité du corps aux corps des dieux dans l'Antiquité, Brest, p. 165-175.

Estienne S., Jaillard D., Lubtchansky N. et Pouzadoux C. éd. (2008), Image et religion dans l'Antiquité gréco-romaine, Naples.

Estienne S., Huet V., Lissarrague F. et Prost F. éd. (2014), Figures des dieux. Construire le divin en images dans l'Antiquité, Rennes.

Estienne S. et Lissarrague F. (2015), « Le corps des dieux dans les mondes grecs et romains : bilan historiographique ", dans Gherchanoc F. éd., L'histoire du corps dans l'Antiquité : bilan historiographique, DHA suppl. 14, p. 19-29.

Fales F. M. (2004), «Rileggendo gli inventari di Qatna », Kaskal : rivista di storia, ambiente e culture del Vicino Oriente Antico 1, p. 83-127.

Gaifman M. (2012), Aniconism in Greek Antiquity, Oxford.

Gaspa S. (2014), « Golden Decorations in Assyrian Textiles, An Interdisciplinary Approach », dans Harlow M., Michel C. et Nosch M.-L. éd., Prehistoric, Ancient Near Eastern and Aegean Textiles and Dress, an Interdisciplinary Anthology, Ancient Textiles Series 18, Oxford, p. 227-244.

Gaspa S. (2018), Textiles in the Neo-Assyrian Empire: A Study of Terminology, SANER 19, Berlin.

Gherchanoc F. (2014), « Le jugement de Pâris et les jeux de vêtement dans la céramique attique aux $\mathrm{VI}^{\mathrm{e}}$ et $\mathrm{V}^{\mathrm{e}}$ siècles avant notre ère ", dans Huet V. et Gherchanoc $\mathrm{F}$. éd., De la théâtralité du corps aux corps des dieux dans l'Antiquité, Brest, p. 125-141.

Gros P. (2006), « Ornamentum chez Vitruve. Le débat sur le décor architectural à la fin de l'époque hellénistique », dans Gros P., Vitruve et la tradition des traités d'architecture : fabrica et ratiocinatio, Rome, p. 389-398.

Huet V. (2014), « À propos de figures de dieux dans l'Antiquité grecque et romaine, ou comment (et pourquoi) rendre visible l'invisible », Perspective 2, p. 261-266.

Huet V. et Gherchanoc F. éd. (2014), De la théâtralité du corps aux corps des dieux dans l'Antiquité, Brest.

Joannès F. (1992), «Les temples de Sippar et leurs trésors à l'époque néo-babylonienne », Revue d'assyriologie et d'archéologie orientale 86-2, p. 159-184.

Joannès F. (2020), « The Goddess Nanaja's New Clothes », dans Harlow M., Michel C. et Quillien L. éd., Textiles and Gender in Antiquity, Londres, p. 31-42.

Joannès F., Kepinski C. et Lecomte O. (1983), « Présence babylonienne dans le pays de Suhu au XVII ${ }^{\mathrm{e}}$ siècle av. J.-C. : l'exemple de Khirbet ed Diniye (Irak) », Revue d'assyriologie et d'archéologie orientale 77-2, p. 119-142.

Lanoë C. (2013), « Le système de parure comme langage technique », dans Lanoë C. et Moulinier L. éd., Corps parés, corps parfumés, Artefact. Techniques, histoire et sciences humaines 1, p. 13-31.

Leemans W. F. (1952), Ishtar of Lagaba and her Dress, Leyde. 
Leichty E. (2011), The Royal Inscriptions of Esarhaddon, King of Assyria (680-669 BC), Winona Lake, Indiana.

Limet H. (1960), Le travail du métal au pays de Sumer au temps de la IIIe dynastie d'Ur, Liège.

Maggio M. (2012), L'ornementation des dieux à l'époque paléo-babylonienne. Étude du matériel ayant appartenu aux dieux d'après les documents de la pratique, réflexion sur le don, l'ornementation des statues divines et la conservation des objets précieux, AOAT 393, Münster.

Malamoud Ch. et Vernant J.-P. dir. (1986), Corps des dieux, Le temps de la réflexion VII, Paris.

Michel C. (2006) « Les médaillons solaires dans la documentation paléo-assyrienne : des bijoux pour les dieux ", dans Patrier J., Quenet P. et Butterlin P. éd., Mille et une empreintes. Un Alsacien en Orient. Mélanges en l'honneur du 65e anniversaire de D. Beyer, Thurnhout, p. 319-329.

Moussy C. (1996), « Ornamentum et ornatus : de Plaute à la Vulgate », REL 74, p. 92-107.

Muller V. (2015), « Les tablettes cunéiformes des premières fouilles de Tell Mishrifeh/Qațna : état de la question ", dans Pfälzner P. et Al-Maqdissi M. éd., Qatna and the Networks of Bronze Age Globalism. Proceedings of an International Conference in Stuttgart and Tübingen in October 2009, Qatna Studien Supplementa 2, Wiesbaden, p. 223-228.

Mylonopoulos J. éd. (2010), Divine Images and Human Imaginations in Ancient Greece and Rome, LeydeBoston.

Oppenheim A. L. (1949), « The Golden Garments of the Gods », JNES 8-3, p. 172-193.

Owen D. I. (2019), « A New Iri-Saĝrig “Sacristy” Inventory Text in the Lanier Theological Library ", Revue d'assyriologie et d'archéologie orientale 113-1, p. 39-44.

Paoletti P. (2012), Der König und Sein Kreis das Staatliche Schatzarchiv der III. Dynastie von Ur, BPOA 10, Madrid.

Parpola A. (1985), The Sky-Garment, A Study of the Harappan, and its Relation to the Mesopotamian and Later Indian Religion, Helsinki.

Pironti G. (2014), « Du voile à la voile : réflexions sur l'Aphrodite en voyage et ses parures », dans Huet V. et Gherchanoc F. éd., De la théâtralité du corps aux corps des dieux dans l'Antiquité, Brest, p. 91-106.

Platt V. (2011), Facing the Gods: Epiphany and Representation in Graeco-Roman Art, Literature and Religion, Cambridge.

Pongratz-Leisten B. et Sonik K. éd. (2015), The Materiality of Divine Agency, SANER 8, Berlin.

Prêtre C. (2012), Kosmos et kosmema. Les offrandes de parures dans les inscriptions de Délos, Kernos suppl. 27, Liège.

Prost F. (2008), «L'odeur des dieux en Grèce ancienne. Encens, parfums et statues de culte », dans Bodiou L., Frère D, Mehl V. dir., Parfums et odeurs dans l'Antiquité, Rennes, p. 97-103.

Römer Th., Gonzalez H. et Marti L. éd. (2019), Représenter dieux et hommes dans le Proche-Orient ancien et dans la Bible, OBO 287, Louvain.

Sack R. H. (1977), « Some Remarks on Jewelry Inventories from Sixth Century B.C. Erech », Zeitschrift für Assyriologie 69, p. 41-46.

Sebaï M. (2014), « Orner les dieux en Afrique romaine. Dédicants ou Divinités ? Gestes de pietas sur quelques stèles d'Afrique romaine ", dans Huet V. et Gherchanoc éd., De la théâtralité du corps aux corps des dieux dans l'Antiquité, Brest, p. 177-204. 
Sheer T. S. (2000), Die Gottheit und ihr Bild: Untersuchungen zur Funktion griechischer Kultbilder in Religion und Politik, Munich.

Steiner D. (2001), Images in Mind: Statues in Archaic and Classical Greek Literature and Thought, Princeton.

Vernant J.-P. (1990), Figures, idoles et masques, Paris.

Walker C.B.F. et Dick M. (2001), The Induction of the Cult Image in Ancient Mesopotamia. The Mesopotamian Mīs Pî Ritual, SAALT 1, Helsinki.

Winter I. (2012), « GOLD! Divine Light and Lustre in Ancient Mesopotamia », dans Matthews R., Curtis J. éd., Proceedings of the $7^{\text {th }}$ International Congress on the Archaeology of the Ancient Near East, 12-16 April 2010, the British Museum and UCL, London. Volume 2: Ancient \& Modern Issues in Cultural Heritage Colour \& Light in Architecture, Art \& Material Culture Islamic Archaeology, Wiesbaden, p. 153-171.

Zawadzki S. (1985), « The Foundry of the Neo-Babylonian Temple », EOS Commentarii Societatis Philologae Polonorum 73, p. 101-130.

Zawadzki S. (1991), « Ironsmiths, Bronzesmiths and Goldsmiths in the Neo-Babylonian Texts from Sippar: Contributions to Studies on Babylonian Society in the Second Half of First Millenium B.C. ", Die Welt des Orients 22, p. 21-47.

Zawadzki S. (2006), Garments of the Gods. Studies on the Textile Industry and the Pantheon of Sippar according to the Texts from the Ebabbar Archive, volume 1, Fribourg.

Zawadzki S. (2013), Garments of the Gods. Texts, vol. 2, Fribourg.

\section{NOTES}

1. Voir également, Bodiou et Mehl 2019.

2. Dictionnaire de la langue française. Lexis, Larousse, Paris, 1994 [1979], s. v. "parure ", p. 1335.

3. Voir Lanoë 2013, p. 13-14 et 19.

4. Leichty 2011, p. 103-109 - RINAP 4, $48: 88-89$.

5. Sur l'entretien, la conservation et l'embellissement d'effigies divines par le biais d'onguents et de parfums, voir Prost 2008, p. 101-102.

6. Plutarque, Démétrios, 10, 5 ; 12, 3-4 (trad. R. Flacelière et É. Chambry, CUF, 1977).

7. Cette question est précisément posée, puis analysée à partir de la documentation épigraphique à Rome par Estienne 2014, p. 166.

8. Articles rassemblés dans Vernant 1990. Concernant l'antiquité grecque et romaine, à titre d'exemple, voir le colloque Agalma ou les figurations de l'invisible, Dugast et al. 2021, également Platt 2011, ainsi que les travaux du Groupe de Recherche Européen FIGVRA, La représentation du divin dans les mondes grecs et romains piloté par Nicole Belayche, notamment l'ouvrage conclusif Belayche et Pirenne-Delforge 2015 ; voir aussi les bilans historiographiques de Huet 2014; Estienne et Lissarrague 2015. Concernant la Mésopotamie, voir Cassin 1968 et plus récemment Walker et Dick 2001 ; Asher-Greve et Westenholz 2013 ; Pongratz-Leisten et Sonik 2015 ; Römer et al. 2019. 
9. $A H w$, p. 1266-1267; CAD Š/III, p. 237-238, šukuttu A. En médio-assyrien et néoassyrien, le terme dumāqu désigne aussi les bijoux, dont ceux des divinités ( $A H w$, p. 175-176; CAD D, p. 176) et à l'époque néo-babylonienne, le terme taškuttu ( $A H w$, p. 1338 ; CAD T, p. 290).

10. AHw, p. 1360-1361 «ordentliche Ausstattung; Schmuck»; CAD T, p. 422-423 « embellishment, ornament, insigna ».

11. Leichty 2011, p. 137 - RINAP 4, 60 : 40’-41', p. 137 et Borger 1956, p. 88 : 15-16.

12. AHw, p. 1473-1474 sous (w)asāmu(m) ; CAD A/II, p. 328-329, asāmu 1 : «to be fitting, proper, suitable». Le terme sumérien hé.du ${ }_{7}$ désigne un ornement, il est issu de ce même verbe $\mathrm{du}_{7}(a s \bar{a} m u)$.

13. AHw p. 1046 simtu 3a ; CAD S, p. 281, simtu 31 '.

14. $A H w$ p. 643 «Schreckensglanz(maske)»; CAD M, p. 9, melammu « radiance, supernatural awe-inspiring sheen, glow of good health». Voir Cassin 1968; Winter 2012. Par extension, le melammu peut aussi émaner des démons et des rois ou même d'objets divinisés ou bien de temples.

15. Cassin 1968, p. 24.

16. Voir Casevitz 1989 ; Cartledge et al. 1998 ; Colburn et Heyn 2008; Briand 2011 ; Prêtre 2012.

17. Bodiou et al. 2011, p. 2.

18. Estienne 2014, p. 165-166 ; voir également Moussy 1996 et Gros 2006.

19. Estienne 2014, p. 166.

20. Voir, en premier lieu, Malamoud et Vernant 1986.

21. Voir Estienne et al. 2008 ; Mylonopoulos 2010 ; Borgeaud et Fabiano 2013 ; Estienne et al. 2014. Voir également Sheer 2000 ; Bettinetti 2001 ; Steiner 2001 ; Gaifman 2012.

22. Voir Pironti 2014 ; Gherchanoc 2014.

23. Voir Huet et Gherchanoc 2014, plus particulièrement les parties 2 et 3 de l'ouvrage.

24. Brouillet et Carastro 2018.

25. Voir Prost 2008, p. 98-99 et 101-102 ; Estienne 2014 ; Sebaï 2014.

\section{AUTEURS}

\section{FLORENCE GHERCHANOC}

Université de Paris, ANHIMA UMR 8210

LOUISE QUILLIEN

CNRS, ArScAn UMR 7041 